\title{
La enfermedad periodontal materna como factor de riesgo del retardo de crecimiento intrauterino
}

The maternal periodontal disease as a risk factor of intrauterine growth retardation

\section{Resumen}

El estudio evalúa la relación entre retardo de crecimiento intrauterino (RCIU) y la enfermedad periodontal (EP) materna. Este estudio tipo casos y controles de 120 madres, 38 casos (madres de bebés con RCIU) y 82 controles (madres de bebés sin RCIU) se efectuo en el Hospital Docente Madre Niño San Bartolomé. Se interrogó a las madres y se revisaron las historias clínicas. Se registraron datos como: grado de instrucción, estado civil, hábitos de fumar, alcohol y drogas. Todas se examinaron dentro de los dos días posteriores al parto, un solo examinador evaluó los componentes de placa, gingival y periodontal mediante el índice de enfermedad periodontal (IEP) modificado que observa la profundidad al sondaje, nivel de inserción clínica, sangrado al sondaje, inflamación gingival y presencia de placa. Se calculó la distribución de frecuencias y la prueba de Odds Ratio. Se encontró que la enfermedad periodontal materna aincrementan ligeramente la probabilidad de tener nińos con RCIU [condiciones de placa OR 1,49 (IC 95\% 0,13-1,96), gingivales OR 1,36 (IC 95\% 1,06-1,74) y periodontales OR 1,32 (IC $95 \% 0,09-1,82)]$. Se concluye que la enfermedad periodontal materna no representa un factor de riesgo perceptible del retardo del crecimiento intrauterino.

Palabras clave: Enfermedad periodontal, retardo del crecimiento fetal, factores de riesgo de RCIU.

\section{Abstract}

This study evaluates the relationship between the intrauterine growth retardation (IUGR) and the maternal periodontal disease (MPD). This case-control study of 120 mothers, 38 cases (mothers of babies with IUGR) and 82 controls (mothers of babies without IUGR) at the "Madre Niño San Bartolomé" Teaching Hospital. The mothers were interrogated and their medical histories were reviewed. Data was recorded, such as: the education level, marital status, smoking, alcohol and drug habits. All the mothers were examined within two days after the delivery. Only one examiner evaluated the plaque, gingival and periodontal components through the modified periodontal disease index (PDI) that observes the probing depth, clinical attachment level, proving bleeding, gingival inflammation and plaque presence. The frequency distribution and the Odds Ratio test were calculated. We found out that the maternal periodontal disease increases a little the probability of having children with IUGR [plaque conditions OR 1.49 (IC 95\% 0.13-1.96), gingival conditions OR 1.36 (95\% IC 1.06-1.74) and periodontal conditions OR 1.32 (IC 95\% 0.09-1.82)]. It can be concluded that the maternal periodontal disease does not represent a substantial risk factor for intrauterine growth retardation.

Key words: Periodontal disease, fetal growth retardation, IUGR risk factors.

\section{Introducción}

El retardo del crecimiento intrauterino (RCIU) es una de las principales causas de morbimortalidad perinatal en el mundo. ${ }^{1}$ En nuestro país, el índice de RCIU se calcula entre 1 y $7 \%$; por otro lado, en países más desarrollados solo llega de 1 a $3 \%$. $^{2,3}$

El diagnóstico de RCIU es de acuerdo a un peso al nacer por debajo del décimo percentil de la distribución de peso según edad gestacional; siendo considerados como RCIU aquellos neonatos cuyo peso está por debajo del décimo percentil del peso que le corresponde para su edad gestacional. ${ }^{4}$

El RCIU tiene un especial interés en salud pública, ya que se asocia a mayor riesgo de morbilidad, mortalidad, alteraciones inmunológicas, malforma- ciones congénitas y secuelas neurológicas; y es un indicador de desnutrición, pobreza, y deficiente conocimiento en atención en salud. ${ }^{5-7}$ Sin embargo, hay la posibilidad de que existan otros factores de riesgo, más aún cuando diferentes estudios encuentran que las madres de nacidos con bajo peso o RCIU tienen una pobre salud periodontal. ${ }^{8-17}$

Según estas investigaciones, la EP materna al ser una enfermedad infecciosa que afecta los tejidos periodontales es susceptible a las variaciones fisiológicas en los niveles de hormonas esteroideas que se presentan en el embarazo. Esta alteración, al ser exagerada, incrementa a su vez los niveles de PGE2 y TNF, los cuales parecen determinar la magnitud de la respuesta del crecimiento fetal. ${ }^{12,18}$

En base a lo anterior, es necesario identificar los factores de riesgo de RCIU para
Artículo Original

\section{Maynor Carranza Samanez ${ }^{1}$ Sixto García Linares² Julissa Dulanto Vargas ${ }^{3}$}

\author{
Práctica privada. \\ ${ }^{2}$ Profesor Asociado del D.A. Médico Quirúrgico \\ Facultad de Odontología de la UNMSM. \\ *Tesis para optar el título de Cirujano Dentista
}

Correspondencia:

C.D. Kilder Maynor Carranza Samanez

Av. Cipriano Dulanto 1096, Lima 21. Perú

Teléfono: 9-9466-9074

Correo electrónico: kildermaynor@hotmail.com disminuir su incidencia, considerando que el neonato con RCIU presenta una elevada tasa de morbimortalidad.

El objetivo del estudio fue determinar la relación de la enfermedad periodontal (EP) en las gestantes, como factor de riesgo de RCIU en el Hospital Docente Madre Niño San Bartolomé (HODOMANI).

\section{Material y Método}

Es un estudio de casos y controles. La población la conformaron las madres que dieron a luz en el Servicio de GinecoObstetricia del Hospital Docente Madre Niño San Bartolomé durante el periodo de agosto a octubre de 2008. Para los criterios de inclusión, se seleccionaron madres de recién nacidos vivos únicos que aceptaron participar del estudio y con el 
consentimiento informado, con más de 10 piezas dentarias en boca y sin profilaxis antibiótica. La muestra la conformaron 38 casos (recién nacido con RCIU) y 82 controles (recién nacido sin RCIU).

El criterio diagnóstico de RCIU se realizó después del alumbramiento considerando para ello la tabla de referencia de Williams, todos los recién nacidos con un peso inferior al percentil $10 \mathrm{del}$ peso correspondiente a su edad gestacional fueron considerados en el grupo de $\operatorname{casos}^{19}$.

La evaluación periodontal consideró el índice de enfermedad periodontal (IEP) modificado que toma en cuenta los siguientes indicadores: profundidad al sondaje, nivel de inserción clínica, sangrado al sondaje, inflamación gingival y placa blanda en las piezas dentarias: 16 , 21, 24, 36, 41 y 44.

El examen clínico periodontal se realizó dentro de las 48 horas posteriores al parto, realizado por un solo examinador y con las madres sentadas en su cama en el hospital e iluminación de una lámpara frontal.

Primero. Se evaluó la condición periodontal con una sonda periodontal Hu Friedy (PCP 15) para observar la profundidad al sondaje (distancia del margen gingival hasta donde ingrese la sonda periodontal), nivel de inserción clínica (distancia del límite amelocementario a la profundidad al sondaje). Se midieron en $\mathrm{mm}$ en 2 zonas por diente: una por vestibular y una por palatino; se clasificaron numéricamente de acuerdo al IEP ( $4=$ nivel de inserción $\geq 1 \mathrm{~mm} \leq 3 \mathrm{~mm}, 5=$ nivel de inserción $>3 \mathrm{~mm}$ hasta $6 \mathrm{~mm}$ hacia apical, $6=$ nivel de inserción $>6 \mathrm{~mm}$ ). Para hallar la valoración de la condición periodontal todos los puntajes obtenidos eran sumados y divididos entre el número de piezas examinadas.

Segundo. Se evaluó la condición gingival tomando en cuenta la inflamación y sangrado, observadas únicamente en aquellas pacientes con niveles de inserción menores a $1 \mathrm{~mm}$. La observación se realizó en 4 zonas del diente: tres por vestibular (mesial, medio y distal) y una por palatino; se registraron los promedios de los valores por paciente y el puntaje criterio de gingivitis ( 0 = ausencia, 1 = leve, 2 = moderada y 3 = severa).

A todas las pacientes se evaluó la condición de placa blanda en 2 zonas del diente: vestibular y palatino, y se registraron los promedios de los valores por paciente y el puntaje criterio de placa (ausencia, menos de $1 / 3$, hasta $2 / 3$, más de $2 / 3$ ).

Tabla 1. Características socioeconómicas de la muestra. Hospital Docente Madre Nińo San Bartolomé. 2008

\begin{tabular}{lccc}
\hline VARIABLE & $\begin{array}{c}\text { CASOS } \\
(\mathbf{n}=\mathbf{3 8}) \%\end{array}$ & $\begin{array}{c}\text { CONTROLES } \\
(\mathbf{n}=\mathbf{8 2}) \%\end{array}$ & Valor-p \\
\hline Edad materna & 11 & & \\
13 a 18 años & 26 & 36 & $>0,05 \mathrm{~h}$ \\
19 a 24 años & 13 & 24 & \\
25 a 30 años & 39 & 13 & \\
31 a 36 años & 11 & 16 & \\
Mayor 37 años & & & $>0,05$ \\
Estado civil & 21 & 16 & \\
Soltera & 16 & 17 & \\
Casada & 63 & 67 & $>0,05$ \\
Conviviente & & & \\
Grado de instrucción & 5 & 44 & \\
Primaria & 63 & 22 & \\
Secundaria & 32 & & \\
Superior & & & \\
\hline
\end{tabular}

Las covariables fueron: Edad, talla y peso materno; grado de instrucción, estado civil, consumo de: cigarrillo, controles prenatales, antecedentes de aborto o bajo peso, condiciones del embarazo actual (estado nutricional), condiciones asociadas y diagnóstico (infección urinaria, preclampsia).

Tabla 2. Características biológicas y antecedentes gestacionales. Hospital Docente Madre Niño San Bartolomé. 2008

\begin{tabular}{|c|c|c|c|}
\hline VARIABLE & $\begin{array}{c}\text { CASOS } \\
(n=38) \%\end{array}$ & $\begin{array}{l}\text { CONTROL } \\
(n=82) \%\end{array}$ & Valor $-\mathrm{P}$ \\
\hline \multicolumn{4}{|l|}{ Peso del recién nacido } \\
\hline Menos de $2500 \mathrm{~g}$ & 94,7 & 86,6 & \multirow[t]{2}{*}{$>0,05$} \\
\hline Mayor o igual a $2500 \mathrm{~g}$ & 5,3 & 13,4 & \\
\hline \multicolumn{4}{|l|}{ Edad gestacional } \\
\hline Pretérmino & 60,5 & 36,6 & \multirow[t]{2}{*}{$<0,05$} \\
\hline A término & 39,5 & 63,4 & \\
\hline \multicolumn{4}{|l|}{ Estado nutricional materno } \\
\hline Bajo peso & 68,4 & 28 & \multirow{4}{*}{$<0,05$} \\
\hline Adecuado peso & 29 & 58,6 & \\
\hline Sobrepeso & 0 & 8,6 & \\
\hline Obesidad & 2,6 & 4,8 & \\
\hline Antecedente aborto & 46,9 & 23,2 & $<0,05$ \\
\hline Antecedente parto prematuro & 28,1 & 18,3 & $<0,05$ \\
\hline Infección urinaria & 18,8 & 20,7 & $<0,05$ \\
\hline \multicolumn{3}{|l|}{ Control prenatal } & \multirow{3}{*}{$>0,05$} \\
\hline Menor igual 4 & 28,9 & 28,0 & \\
\hline Mayor 4 & 71,1 & 72,0 & \\
\hline \multicolumn{4}{|l|}{ Paridad } \\
\hline Primípara & 50 & 54,9 & \multirow[t]{2}{*}{$>0,05$} \\
\hline Multípara & 50 & 45,1 & \\
\hline
\end{tabular}

Los datos de la evaluación periodontal fueron grabados y transcritos a la ficha de recolección de datos. El análisis estadístico incluyó la comparación de los valores promedio de cada una de las variables según casos y controles. Luego, se procedió a estimar el Odds Ratio (OR), que indicase la asociación de las variables de EP con la variable RCIU. 


\section{Resultados}

La muestra estuvo conformada por 120 madres. El promedio de edad de los casos fue 28 años y 27,4 años para los controles.

En la Tabla 1, se observa la distribución de la muestra de estudio según características socioeconómicas. No se encontraron diferencias entre los grupos en cuanto a grupo etáreo, grado de instrucción o estado civil ( $p>0,05)$. Ninguna mujer declaró fumar diariamente, consumir más de una bebida alcohólica por semana, ni tener el hábito de ser consumidor de drogas.

En la Tabla 2, observamos que el 94,7 $\%$ de los casos tuvieron un peso inferior a $2500 \mathrm{~g}$; sin embargo, solo el 60,5\% fueron nacidos pretérmino. Con respecto a la distribución de la variable edad gestacional, se observó una distribución inversa entre casos y controles,

Tabla 3. Índice de enfermedad periodontal modificado, Hospital Docente Madre Niño San Bartolomé. 2008

\begin{tabular}{|c|c|c|c|}
\hline VARIABLE & $\begin{array}{c}\text { CASOS } \\
\%\end{array}$ & $\begin{array}{c}\text { CONTROL } \\
\%\end{array}$ & Valor-p \\
\hline Criterio de placa blanda & $(n=38)$ & $(n=82)$ & \multirow{5}{*}{$<0,05$} \\
\hline Ausencia de placa & 5,3 & 13,4 & \\
\hline Menos de $1 / 3$ hasta $2 / 3$ de placa & 2,6 & 15,9 & \\
\hline Hasta $2 / 3$ de placa & 26,3 & 35,4 & \\
\hline Más de $2 / 3$ de placa & 65,8 & 35,4 & \\
\hline Criterio gingival y periodontal & $(n=38)$ & $(n=82)$ & \multirow{8}{*}{$<0,05$} \\
\hline $0=$ Ausencia de inflamación & 2,6 & 6,1 & \\
\hline 1 = Inflamación leve & 7,9 & 30,5 & \\
\hline 2 = Inflamación moderada & 26,3 & 26,8 & \\
\hline 3 = Inflamación severa & 21,1 & 12,2 & \\
\hline $4=$ Nivel de inserción $\geq 1 \mathrm{~mm} \leq 3 \mathrm{~mm}$ & 21,1 & 8,5 & \\
\hline $5=$ Nivel de inserción $>3 \mathrm{~mm}$ hasta $6 \mathrm{~mm}$ & 13,2 & 12,2 & \\
\hline $6=$ Nivel de inserción $>6 \mathrm{~mm}$ & 7,9 & 3,7 & \\
\hline
\end{tabular}

Tabla 4. Riesgo de índice de enfermedad periodontal modificado, Hospital Docente Madre Niño San Bartolomé. 2008

\begin{tabular}{|c|c|c|c|c|c|}
\hline VARIABLE & $\begin{array}{c}\text { CASOS } \\
\%\end{array}$ & $\begin{array}{c}\text { CONTROL } \\
\%\end{array}$ & $\mathbf{P}$ & Riesgo & IC $95 \%$ \\
\hline Criterio de placa blanda & $(n=38)$ & $(n=82)$ & & & \\
\hline Hasta $2 / 3$ de placa & 34,2 & 64,6 & 0,002 & 1,49 & $0,13-1,96$ \\
\hline Más de $2 / 3$ de placa & 65,8 & 35,4 & & & \\
\hline Criterio gingival & $(n=22)$ & $(n=60)$ & & & \\
\hline Gingivitis ausente y leve & 18,25 & 46,7 & 0,02 & 1,36 & $1,06-1,74$ \\
\hline $\begin{array}{l}\text { Gingivitis moderada y } \\
\text { severa }\end{array}$ & 81,8 & 53,3 & & & \\
\hline Criterio periodontal & $(n=38)$ & $(n=82)$ & & & \\
\hline Nivel de inserción $<1 \mathrm{~mm}$ & 57,9 & 75,6 & 0,05 & 1,32 & $0,09-1,82$ \\
\hline Nivel de inserción $\geq 1 \mathrm{~mm}$ & 42,1 & 24,4 & & & \\
\hline
\end{tabular}

\section{Discusión}

Ninguna mujer presentó diabetes, alteración úteroplacentaria, ni antecedente de enfermedad de trasmisión sexual.

En la Tabla 3, muestra las comparaciones entre casos y controles según índice de enfermedad periodontal. Los criterios de placa blanda, gingival y periodontal mostraron diferencias significativas, se observó tendencia de los casos a una peor condición periodontal que los controles.

En la Tabla 4, se observa la asociación entre las condiciones de enfermedad periodontal de las madres y RCIU de los nińos; los resultados muestran ligeramente aumentado el riesgo de las condiciones periodontales para RCIU: placa blanda OR: 1,49 (IC $95 \% 0,13$ - 1,96); condición gingival OR: 1,36 (IC 95\% 1,06 - 1,74) y condición periodontal OR: 1,32 (IC 95 \% 0,09$1,82)$.

Existen múltiples estudios que asocian la enfermedad periodontal con efectos adversos en el embarazo. ${ }^{8-12,18-20}$ Estos afectos se han centrado en el término del embarazo (partos prematuros) y en el peso del nacido (bajo peso al nacer). Recién, en la última década, los estudios vienen considerando al retardo de crecimiento intrauterino como un factor de riesgo. ${ }^{13,17}$

Existe una fuerte asociación entre la prematurez y el bajo peso. Sin embargo, estos dos parámetros no son completamente concordantes, pues hay prematuros nacidos con adecuado peso y nacidos a término con bajo peso. En base a ello, se toma en consideración la restricción del crecimiento intrauterino que refleja de forma más adecuada, el verdadero crecimiento del feto en formación.

El crecimiento del feto es estudiado en modelos con animales para comprobar la asociación con enfermedad periodontal. Dichos estudios demuestran la disminución del crecimiento fetal al producirse una infección lejana al útero por microorganismos periodontopatógenos como Porphyromonas gingivalis. ${ }^{13}$, ${ }^{15}$ En base a dichas evidencias, se consideró pertinente evaluar a la EP materna como factor de riesgo de RCIU.

Aún no hay consenso de definición exacta de EP, requisito indispensable para un criterio uniforme global $^{21}$; sin embargo, la evaluación periodontal se basó en 2 de los parámetros más comunes usados para caracterizar a un paciente con periodontitis: NIC (considerado como el "gold Standard" de la medida de la destrucción periodontal) y PS14, 22, 23 inflamación gingival, sangrado al sondaje y placa evaluados mediante el IEP. ${ }^{24,25}$

Existen factores de confusión para RCIU, la edad materna es una de ellas; sin embargo, el grupo de estudio fue bastante homogéneo y no se encontró en asociación con RCIU; ;,9,23 igualmente sucedió con la distribución madres con infección urinaria, menor número de control prenatal y primíparas. El consumo de alcohol, cigarrillos y drogas, diabetes, alteración úteroplacentaria, también aceptados como factores de riesgo, fueron excluidos del estudio.

El peso materno de los casos resultó menor; probablemente, refleja el bajo peso al inicio del embarazo o la poca ganancia de peso durante la gestación, los cuales son considerados como factor de riesgo durante el embarazo. ${ }^{11,23} \mathrm{El}$ an- 
tecedente de parto prematuro y aborto resultó en un mayor número para los casos, estos también son considerados factores de riesgo de RCIU. ${ }^{20,26}$ Se encontró una relación estadísticamente significativa, igual que López ${ }^{12}$ y Dasanayake.

Todas las madres que ingresaron al estudio provinieron de la misma fuente potencial de sujetos elegibles, madres que dieron a luz en el Hospital Docente Madre Niño San Bartolomé.

La evaluación del IEP mostró diferencias estadísticas significativas $(\mathrm{p}<0,05)$ entre los grupos, con mayor tendencia de las madres de niños con RCIU a mayor presencia de placa, inflamación y pérdida del nivel de inserción clínica, igual que en el estudio de Offenbacher, ${ }^{13}$ Dasanayake, ${ }^{9}$ López $^{12}$ y de Lin. ${ }^{15}$ Por otra parte, difiere de algunos estudios que no han encontrado relación. ${ }^{27,28}$ Es posible que la discrepancia sea por diferencias en la definición de EP, así como casos, ya que en algunos estudios consideran los componentes del RCIU por separado. ${ }^{14}$

\section{Conclusión}

La pérdida de inserción clínica materna incrementa en 1,32 la posibilidad de tener nińos con retardo de crecimiento intrauterino.

La inflamación gingival materna incrementa en 1,36 la posibilidad de tener nińos con retardo de crecimiento intrauterino.

La placa blanda bacteriana materna incrementa en 1,49 la posibilidad de tener nińos con retardo de crecimiento intrauterino.

Los resultados encontrados muestran que la enfermedad periodontal materna aumenta levemente la posibilidad de nacimientos de niños con retardo de crecimiento intrauterino, sin embargo, no es un factor de riesgo perceptible de ello.

\section{Referencias Bibliográficas}

1. Pacheco J. Retardo de crecimiento intrauterino. Ginecología y Obstetricia [serial on line] 1995; 41(2):1827. Disponible en: URL: http:// sisbib.unmsm.edu.pe/BVRevistas/ ginecologia/Vol_41N2/retardo_ de_crecimiento_intrauter.htm

2. Acosta M. Curva de crecimiento intrauterino [Tesis doctoral]. Lima:
Universidad Nacional Mayor de San Marcos; 1972.

3. ACOG Committee on Practice Bulletins-Obstetrics. ACOG Practice Bulletin N.o 80: premature rupture of membranes. Clinical management guidelines for obstetrician-gynecologists. Obstet Gynecol 2007; 109: 1007-19.

4. Pollitt E. Consecuencias de la desnutrición en el escolar peruano. Lima: Pontificia Universidad Católica del Perú.Cap.VI; 2002: 12956.

5. Klebanov PK, Brooks-Gunn J, McCarton C, McCormick MC. The contribution of neighborhood and family income to developmental test scores over the first three years of life. Child development 1998; 69: 1420-36.

6. Caulfield L, Haas J, Belizán J, Rasmussen K, Edmonston B. Differences in early postnatal morbidity risk by pattern of fetal growth in Argentina. Paediatr Perinat Epidemiol 1991; 5: 263-75.

7. Pérez-Escamilla R, Pollit E. Causas y consecuencias de retraso del crecimiento intrauterino en América Latina. Bol Of Sanit Panam 2004; 112: 473-82.

8. Offenbacher S, Katz V, Fertik G, Collins J, Boyd D, Maynor G et al. Periodontal infection as a possible risk factor for preterm low birth weight. J Periodontol 1996; 67:1103-07.

9. Dasanayake AP, Russell S, Boyd D, Madianos PN, Foster T, Hill E. Preterm low birth weight and periodontal disease among africa americans. Dent Clin North Am 2003; 47: 115-25.

10. Cabrales JA, Saenz L, Grau MA, Rojas L, Gonzáles Y, Pina N y Lizano M. Factores de riesgo de bajo peso al nacer en un hospital cubano 1997-2000. Rev Panam Salud Pública 2002; 12: 180-4.

11. Offenbacher S, Jared HL, O’Reilly PG, Wells SR, Salvi GE, Lawrence $\mathrm{HP}$ et al. Potential pathogenic mechanisms of periodontitis-Associated pregnancy complications. Ann Periodontol 1998; 3: 233-48.

12. López NJ, Smith PC, Gutierrez J. Periodontal therapy may reduce the risk of preterm low birth weight in women with periodontal disease: a randomized controlled trial. J Periodontol 2002; 73: 911-24.

13. Offenbacher S, Lieff S, Boggess KA, Murtha AP, Madianos PN, Champagne CME et al. Maternal periodontitis and prematurity. Part I: Obstetric outcome of prematurity and growth restriction. Ann Periodontol 2001; 6:164-74.

14. Robles JJ, Salazar F, Proaño D. Enfermedad periodontal como factor de riesgo de retardo del crecimiento intrauterino. Rev. Estomatol. Herediana 2004; 14:27-34.

15. Lin D, Smith MA, Elter J, Champagne C, Downey CL, Beck J, Offenbacher S. Porphyromonas gingivalis infection in pregnant mice is associated with placental dissemination, an increase in the placental Th1/Th2 cytokine ratio, and fetal growth restriction. Infect Immun 2003; 71: 5163-8.

16. Offenbacher S. Clinical maternal periodontal infections, prematurity and growth restriction. Clin Obstet Gynecol 2004; 47: 808-21.

17. Yeo A, Smith M, Dongming L, Riche E, Moore A, Elter J, Offenbacher S. Campylobacter rectus Mediates Growth Restriction in pregnant mice. J Periodontol 2005; 76: 551-7.

18. Collins JG, Windley HW 3rd, Arnold RR, Offenbacher S. Effects of a Porphyromonas gingivalis infection on inflammatory mediator response and pregnancy outcome in hamsters. Infect Immun 1994; 62: 4356-61.

19. Medina J. Prevalencia del retardo del crecimiento intrauterino y su clasificación en el Instituto Materno Perinatal de Lima [tesis maestría]. Lima: Universidad Peruana Cayetano Heredia; 2002.

20. Offenbacher S, Beck J, Lieff S, Slade G. Role of periodontitis in systemic health: spontaneous preterm birth. J Dent Educ 1998; 62: 85258.

21. Gjermo P, Rösing C, Susin C and Opperman R. Periodontal disease in central and south america. Periodontol 2000 2002; 29: 70-8.

22. American Academy of Periodontology. Position paper epidemiology of periodontal disease. J Periodontol 1996; 67: 935-45. 
23. Lizarraga CA, Proaño D. La enfermedad periodontal como factor de riesgo de parto pretérmino y de bajo peso al nacer en el Hospital Nacional Cayetano Heredia 2002-2003. Rev Med Hered 2005; 16: 172-7.

24. American Academy of Periodontology. Position paper. Diagnosis of periodontal disease. Academy report. J Periodontol 2003; 74: 1237 47.

25. Ramfjord S. Indices for prevalences and incidence of periodontal disease. J Periodontol 1959; 30: 51-9.
26. Delgado J, Gómez L, González V, Ramírez B, Vivas J. Asociación entre enfermedad periodontal y algunas alteraciones del embarazo. Revista Estomatología 2006; 14: 17-21.

27. Davenport ES, Williams CE, Sterne JA, Sivapathasundram V, Fearne JM, Curtis MA. The east London study of maternal chronic periodontal disease and preterm low birth weight infants: Study design and prevalence data. Ann Periodontol 1998; 3: 213-21.
28. Sundell TM, Beazley D, Patters MR, Carr TL, Blankenship JA, Mercer BM. Periodontal disease status and preterm birth in high risk women. J Dent Res 2002;81 (spec. issue A): 230 (Abstr. 1749).

Fecha de recepción: 16-7-09

Fecha de aprobación: 10-06-10 